\title{
Epigenetic Mechanisms in the Pathogenesis of Diabetic Retinopathy
}

\author{
Jun Zeng Baihua Chen \\ Department of Ophthalmology, Second Xiangya Hospital, Central South University, Changsha City, PR China
}

\section{Key Words}

Diabetes $\cdot$ Retinopathy $\cdot$ Epigenetics

\begin{abstract}
Diabetic retinopathy (DR), which arises as a result of an increasing incidence of diabetes mellitus, has gradually become a common disease. Due to its complex pathogenesis, the treatment means of DR are very limited. The findings of several studies have shown that instituting tight glycemic control in diabetic patients does not immediately benefit the progression of retinopathy, and the benefits of good control persist beyond the period of good glycemic control. This has led to the concept of persistent epigenetic changes. Epigenetics has now become an increasingly important area of biomedical research. Recently, important roles of various epigenetic mechanisms have been identified in the pathogenesis of diabetes and its complications. The aim of this review is to provide an overview of the epigenetics and epigenetic mechanisms in diabetes and diabetes complications, and the focus is on the emerging evidence for aberrant epigenetic mechanisms in DR.

(c) 2014 S. Karger AG, Basel
\end{abstract}

\section{Introduction}

Diabetes mellitus, an increasingly common chronic metabolic disorder of insulin production or uptake, is gradually becoming a significant issue worldwide. Diabetic retinopathy (DR), which can lead to blindness if left untreated, is one of the most common microvascular complications of diabetes $[1,2]$. The prevalence of DR was $23 \%$ in the diabetic group in mainland China [3]. It has been the leading cause of blindness amongst 16- to 64-year-olds [4]. The pathogenetic mechanism responsible for DR is imperfectly understood, but much of the mechanism is apparently reproduced by experimental diabetes in animals and by chronic elevation of blood galactose in nondiabetic animals. It is becoming increasingly clear that epigenetic mechanisms contribute significantly to diabetes and diabetic complications. Although much insight into the epigenetic mechanisms in diabetic complications such as cardiovascular diseases has been gained, there is little understood about the epigenetic mechanisms in the pathogenesis of DR. We may uncover potential therapeutic targets and treatment options to prevent the continued development of DR if we clarify the epigenetic mechanisms in DR. The aim of this review is to discuss the current understanding of how epigenetic mechanisms contribute to the formation of DR. 


\section{Pathophysiology of DR}

The pathogenesis of the development of DR is extremely complex because of the involvement of multiple interlinked mechanisms leading to cellular damage and adaptive changes in the retina [5]. In the past, retinopathy has been characterized primarily by its microvascular abnormalities, including abnormal hemodynamics, endothelial cell dysfunction, vessel leakage, vascular occlusion and degeneration, and acellular capillary formation [6]. The microvascular dysfunction may result in two main processes: increased capillary permeability and capillary obliteration, which consequently lead to macular edema and retinal neovascularization in DR, respectively. However, recent studies indicate that DR, which can be defined as a form of chronic neurovascular degeneration, is a composite of structural and functional alterations in both microvascular and neuroglial compartments $[7,8]$. The explicit mechanisms by which hyperglycemia initiates the neuronal or vascular alterations in retinopathy have not been completely defined [6, 7]. Because of an inverse relationship between extracellular glucose concentrations and glucose transport, cells cannot be directly susceptible to direct hyperglycemic damage. In contrast, vascular endothelial cells, major targets of hyperglycemic damage, show no significant change in glucose transport rate when glucose concentration is elevated, resulting in intracellular hyperglycemia [9]. There are several supposed mechanisms underlying hyperglycemiainduced diabetic vascular damage in the retina. The majority of publications focus on the 5 major mechanisms: polyol pathway flux, increased formation of advanced glycation end products, increased expression of the receptor for advanced glycation end products and its activating ligands, activation of protein kinase $\mathrm{C}$ isoforms, and overactivity of the hexosamine pathway, but many of these hypotheses have yet to be validated in human studies or clinical trials $[5,10]$. Emerging evidence shows that these metabolic mechanisms are associated with overproduction of reactive oxygen species and depletion of antioxidants in DR [11], and then reactive oxygen species contribute to the DR progression by damaging retinal cells.

Inflammation is another active factor in the pathophysiology of DR. The upregulation of cytokines and other inflammatory mediators, leading to an influx of leukocytes and persistent low-grade inflammation, is supposed to contribute to diabetes-associated damage to the retinal vasculature and retinal neovascularization. Several proinflammatory mediators, such as vascular endothelial growth factor (VEGF), cytokines, nitric oxide, chemokines, eicosanoid, lipids, angiotensin II and the renin-angiotensin system, have been discovered [12]. Increased expression of vasoactive factors and cytokines probably plays an important role in mediating the structural and functional changes in the retina $[13,14]$. Although recent evidence strongly suggests that inflammation is very important in the pathogenesis of early stages of experimental DR [15-17], studies in humans have not found a consistent association between systemic markers of inflammation and retinopathy $[16,18]$. Also, it is still uncertain whether inflammation plays a crucial role in the development and progression of DR in humans.

Genetic factors and key gene mutations have been proposed to explain the pathogenesis of DR. However, increasing evidence suggests that complex interactions between genes and the environment may play a major role in many common human diseases such as diabetes and its complications [19-21]. Notably, chromatin is a crucial interface between the effects of genetics and environment, and the epigenetic posttranscriptional modifications of histone tails in chromatin have been linked to gene transcription. While several studies have identified key biochemical pathways triggered by hyperglycemia and diabetes in target cells related to DR, the role of epigenetic mechanisms is only now becoming apparent. Furthermore, the Diabetes Control and Complications Trial and the follow-up Epidemiology of Diabetes Interventions and Complications studies have shown that instituting tight glycemic control in diabetic patients does not immediately benefit the progression of retinopathy, and the benefits of good control persist beyond the period of good glycemic control (GC) $[22,23]$. This continued development of DR even after achieving good glucose control suggests a metabolic memory of prior glycemic exposure and indicates epigenetic changes in target cells without alterations in gene coding sequences. Exploring a role of epigenetics in DR could allow for new insights clarifying the interplay between the environment and gene regulation and identify much needed new therapeutic targets.

\section{Epigenetics: An Overview}

Epigenetics is the study of heritable changes in gene expression that occur without changes in DNA sequence [24]. Epigenetic mechanisms regulate both long-term (heritable) and short-term (nonheritable) effects and thus have a significant influence on many different disease 
processes [25]. Chromatin is composed of subunits called nucleosomes containing DNA wrapped around a histone octamer with 2 copies each of histone $\mathrm{H} 2 \mathrm{~A}, \mathrm{H} 2 \mathrm{~B}, \mathrm{H} 3$ and $\mathrm{H} 4$ [26]. It is a dynamic process of transcriptional activation or repression, depending on the recruitment of protein complexes that alter chromatin structure via enzymatic modifications of histone tails and nucleosome remodeling. An open chromatin conformation results in a greater accessibility of transcription factors to the DNA, allowing for gene transcription, while a closed chromatin structure prevents transcription. These epigenetic histone posttranslational modifications include lysine acetylation, methylation and phosphorylation. Along with other epigenetic factors such as DNA methylation and noncoding RNAs such as microRNAs (miRNAs), these posttranslational modifications in chromatin form an added layer of gene regulation without altering the DNA code itself. Histone modifications, such as methylation, phosphorylation and acetylation, result in conformational changes of the histones. Histone acetyltransferases (HATs) and histone deacetylases (HDACs) are regulators of histone lysine acetylation which usually occurs quite rapidly [27]. Histone methylation is generally considered to be more long lasting. Histone lysine methylation is mediated by histone methyltransferases (HMTs) and more complex because lysine residues can be mono-, di- or trimethylated. Generally speaking, activation or repression of gene expression depends on the modification, location as well as the residue modified. For example, histone $\mathrm{H} 3$ lysine 4 methylation ( $\mathrm{H} 3 \mathrm{~K} 4 \mathrm{me})$ is generally associated with gene activation, but histone $\mathrm{H} 3$ lysine 9 methylation (H3K9me) with gene repression [28]. Both promoter and coding regions can be modified, adding another level of complexity to gene transcription outcomes. Although histone modifications occur on all histones, the modifications on histones $\mathrm{H} 3$ and $\mathrm{H} 4$ are the most widely characterized. Among the different modifications, the most studied is acetylation. Acetylation is maintained by the actions of HATs and HDACs. Hyperacetylation of lysine residues at the $\varepsilon$-amino group in the $\mathrm{N}$ terminus of histones occurs as a result of the actions of HATs, which normally results in increased gene transcription. Deacetylation by HDACs is normally linked with decreased gene transcription [29]. There are some important positions for histone acetylation, including lysine 9 (K9) and K14 on histone $\mathrm{H} 3$, and $\mathrm{K} 5, \mathrm{~K} 8, \mathrm{~K} 12$ and $\mathrm{K} 16$ on histone $\mathrm{H} 4$ [30].

Another mechanism by which epigenetic changes control gene expression is DNA methylation. DNA methylation is regulated by DNA methyltransferases at the $5^{\prime}$ po-

Epigenetic Mechanisms in the Pathogenesis of DR sition of cytosine residues in $\mathrm{CpG}$ dinucleotides by transferring methyl groups from S-adenosyl methionine. Hypermethylation of promoter $\mathrm{CpG}$ islands generally leads to transcription repression [31]. Histone acetylation can prevent DNA methylation by inhibiting the binding of DNA methyltransferases. Conversely, DNA methylation can promote histone deacetylation and recruitment of the histone methyltransferase SUV39H1 that mediates $\mathrm{H} 3 \mathrm{~K} 9 \mathrm{me} 3$ to further stabilize the repressive status [32]. Evidence also demonstrates the interaction of DNA methylation and other histone modifications [33]. DNA methylation has been extensively studied in the context of tumor suppressor genes and cancer [34]. However, much less is known about DNA methylation in diabetes and its complications. Following cell division, the pattern of DNA methylation in differentiated somatic cells is both stable and heritable. Alterations of DNA methylation can result in changes in the chromatic configuration, making the promoter region either more or less accessible to transcription factors.

Gene and protein expressions are also regulated by miRNA and other small RNAs posttranscriptionally, which have greater temporal flux, making any interpretation variability of these more formidable [35]. miRNAs are short, single-stranded RNA strands which will not translate into protein [36]. Instead, they block gene translation via binding to complementary regions of mRNA. miRNAs are also able to initiate the degradation of mRNA strands to which they are bound [37]. Recent researches suggested a critical role for miRNAs in various diseases. They have been found to play key roles in differentiation, proliferation, development and in cancer, where they can act as tumor suppressors or oncogenes [38-40].

\section{Epigenetic Mechanisms in Diabetes and Diabetic Complications}

Recent researches have implicated an important role for epigenetic histone modifications and histone posttranslational modifications in diabetes and its complications. HATs and HDACs, modulating nuclear factor (NF)- $\kappa \mathrm{B}$ transcriptional activity and resulting in changes in downstream inflammatory gene expression levels, have been found in the regulation of several key genes related to diabetes [41-43]. Interestingly, monocytes cultured in high-glucose medium (HG) revealed increased recruitment of HATs resulting in histone lysine acetylation at key inflammatory gene promoters, with a corresponding increase in gene expression, and there were 
similar phenomena in blood monocytes from type 1 and type 2 diabetes patients [43]. Another study showed that oxidized lipids can result in increased histone acetylation at the inflammatory gene promoter, with a corresponding increase in gene expression [44]. Further studies suggested that HG increased p300, led to increased histone acetylation at promoters of key extracellular cell matrix genes and vasoactive factors in endothelial cells [45]. However, curcumin, as an inhibitor of p300, could prevent hyperglycemia-induced changes in gene expression levels [45, 46]. Studies in diabetic kidneys demonstrated an important role for HDACs in transforming growth factor- $\beta_{1}$ (TGF- $\beta_{1}$ )-mediated extracellular cell matrix production and fibrosis [47]. These results implicate a role for histone acetylation in promoting gene expression correlated with diabetic complications. Except for histone lysine acetylation, H3K4me and recruitment of the HMT SET7/9 also increased in the insulin promoter region, while $\mathrm{H} 3 \mathrm{~K} 9 \mathrm{me}$ was undetectable $[48,49]$. Since histone lysine methylation is relatively more stable, its role in diabetic complications attracts people to explore, and the role of key histone methylation marks in diabetes and its complications needs to be determined. Cultured monocytes treated with HG presented dynamic changes in both the H3K4me2activating mark and $\mathrm{H} 3 \mathrm{~K} 9$ me2-repressive mark, verifying a role for hyperglycemia in altered histone methylation patterns. The involved changes were also seen in monocytes from diabetic patients [50]. A subset of genes in diabetic lymphocytes displayed increased H3K9me2 linked to immune and inflammatory pathways often associated with type 1 diabetes and its complications [51]. These epigenomic profiling studies suggest that, although the histone methylation pattern is sustained reasonably stable in healthy individuals, it can be disrupted in some disease states. The mechanisms which are responsible for aberrant epigenetic histone methylation occurring in diabetic conditions are further elucidated by additional in vitro experiments. In monocytes, the occupancy of the NF- $\kappa B$ p 65 subunit and p 300 HAT at monocyte chemoattractant protein- 1 and TNF- $\alpha$ promoters decreased by knocking out of the H3K4 HMT SET7/9, with a corresponding decreased $\mathrm{H} 3 \mathrm{~K} 4 \mathrm{me}$ at promoter and gene expression, suggesting that SET7/9 might coactivate NF- $\mathrm{KB}$ transcriptional activity by promoter $\mathrm{H} 3 \mathrm{~K} 4 \mathrm{me}$ activation in response to inflammatory stimuli in the diabetic condition [52]. Likewise, a role in regulating NF- $\kappa \mathrm{B}$ expression for SET7/9 in response to HG was also observed in endothelial cells $[53,54]$. Recent evidence has demonstrated that renal mesangial cells treated with TGF- $\beta$ increased HMT SET7/9 occupancy and the associated active $\mathrm{H} 3 \mathrm{~K} 4$ me marks, but reduced repressive $\mathrm{H} 3 \mathrm{~K} 9$ me marks at promoters of key fibrotic genes linked to diabetic nephropathy. Both TGF- $\beta$ and HG induced promoter histone methylation changes, and gene expression reversed by treatment of TGF- $\beta$ antibody [55]. These results suggest a role for histone modification in modulating gene expression under diabetic conditions.

Recently, some studies have begun to explore the role of DNA methylation in diabetes and its complications. In animal models, epigenetic silencing due to increased promoter DNA methylation has been linked to islet dysfunction and development of diabetes [56,57]. A recent report demonstrated that the insulin promoter DNA was methylated in mouse embryonic stem cells and only became demethylated when the cells differentiated into insulinexpressing cells, and both the mouse and human insulin promoters were specifically demethylated in pancreatic $\beta$-cells, suggesting epigenetic regulation of insulin expression [58]. What is more, DNA methylation and expression of the agouti gene can affect the tendency to develop obesity and diabetes in the agouti mouse [59]. A study showed that both histone modifications and DNA methylation were implicated in the process of intrauterine growth retardation leading to type 2 diabetes, which is due to epigenetic silencing of Pdx1, a key transcription factor that regulates insulin gene expression and $\beta$-cell differentiation [56]. In another study, it was shown that increased DNA methylation of the promoter of the peroxisome proliferator-activated receptor- $\gamma$ coactivator $1 \alpha$ gene, a factor that plays a key role in regulating mitochondrial genes and in the modulation of diabetes in diabetic islets [56].

Several studies have implicated an important role of miRNAs in the pathogenesis of diabetes [60-63]. However, the role of miRNAs in diabetes vascular complications is less studied. miRNAs are linked with the regulation of genes relevant to insulin secretion, fat metabolism, cholesterol biosynthesis and adipogenesis, crucial pathways in the pathogenesis of diabetes $[61,64,65]$. miRNAs also play a role in TGF- $\beta$ signaling related to the pathogenesis of diabetic nephropathy, with key miRNAs such as miR-192, miR-216a, miR-217 and miR-377 being upregulated, resulting in increased fibronectin and collagen expression [66-68]. Currently, there is an area of great interest concerning the role of miRNAs and potential relationships to epigenetic mechanisms in diabetic complications. In a word, epigenetic changes at specific target gene promoters might explain the accelerated development of diabetes and diabetic complications.
Zeng/Chen 


\section{Epigenetic Mechanisms in DR}

Recently, the role of epigenetic mechanism in the pathogenesis of diabetic complications is gaining increasing attention [69], and there is more emerging evidence for aberrant epigenetic mechanisms in DR. Poor glycemic control (PC) has a close relation to DR. The retinas and retinal endothelial cells (RECs), from streptozotocin (STZ)-treated rats, kept in PC show increased expression of HDAC1, HDAC2 and HDAC8, and reduced activity of a histone $\mathrm{H} 3$-specific acetyltransferase; these changes were not reversed with the change of $\mathrm{PC}$ returned to good control (GC). It suggests that the epigenetic metabolic memory phenomenon may be the major reason for the continuation of DR even when the blood glucose level returns to normal [70].

Administration of antioxidants or overexpression of sod2 prevents the development of DR in rodents, suggesting a major role in the development of DR. Sod2, a gene encoding manganese superoxide dismutase, undergoes epigenetic regulation, and these modifications fail to reverse after termination of hyperglycemic insult in the pathogenesis of DR [71-73]. Recent evidence demonstrated that epigenetic changes of retinal Sod2 have an important role in the development of DR and in the metabolic memory phenomenon [74, 75]. Hyperglycemia induced increased acetyl H3K9, H4K20me3 and NF- $\kappa B$ p 65 at the promoter and enhancer of retinal sod2, gene expression and upregulated protein of SUV420h2, and increased the interactions of acetyl $\mathrm{H} 3 \mathrm{~K} 9$ and NF- $\kappa \mathrm{B}$ p65 to H4K20me3. These changes were not prevented by reversal of hyperglycemia. SUV420h2, an enzyme important for di- and trimethylation, is increased in the retina and its capillary cells in hyperglycemia. Silencing SUV420h2 prevented H4K20me3 at sod2, which strongly suggests that SUV420h2 is important in the metabolic memory phenomenon associated with DR [74]. Hyperglycemia also reduced $\mathrm{H} 3 \mathrm{~K} 4 \mathrm{me} 1$ and $\mathrm{H} 3 \mathrm{~K} 4 \mathrm{me} 2$, which are regulated by lysine-specific demethylase-1 (LSD-1), and increased the binding of LSD-1 and Sp1 at Sod2. LSD-1 specifically demethylates mono- or dimethylated H3K4 and $\mathrm{H} 3 \mathrm{~K} 9$, and removal of methyl groups from $\mathrm{H} 3 \mathrm{~K} 4$ is related to transcriptional repression [76]. Thus, regulation of LSD-1 by small interfering RNA ameliorated the glucose-induced decrease in $\mathrm{H} 3 \mathrm{~K} 4 \mathrm{me}$ at Sod2, and prevented the decrease in Sod2 gene expression. In rats, return to GC cannot reverse the decrease in $\mathrm{H} 3 \mathrm{~K} 4 \mathrm{me} 1$ and H3K4me 2 at Sod2, and LSD-1 remained active with increased binding of LSD-1 and Sp1 at Sod2 [77]. De-

Epigenetic Mechanisms in the Pathogenesis of DR creased H3K4me2 and increased LSD-1 at Sod2 also presented in retinas from human donors with DR [76].

Epigenetic modifications contribute to the mitochondrial damage and are postulated in the development of $\mathrm{DR}$, and also to the metabolic memory phenomenon [77]. Retinal matrix metalloproteinase-9 (MMP-9), activated in the diabetic milieu, damages the mitochondria and augments capillary cell apoptosis. Decreased H3K9me2 and increased acetyl H3K9 and p65 at the retinal MMP-9 promoter were found in the diabetic condition. The enzyme activity and transcripts of LSD-1 were elevated. The glucose-induced increase in p65 and decrease in $\mathrm{H} 3 \mathrm{~K} 9 \mathrm{me} 2$ at the MMP-9 promoter can be ameliorated by LSD-1 small interfering RNA. LSD-1 small interfering RNA also prevented MMP-9 activation, mitochondrial damage and cell apoptosis [78]. Thus, regulation of MMP9 by epigenetic modifications has an important role in the development of DR.

Thioredoxin-interacting protein (TXNIP) plays a causative role in diabetes and its vascular complications. In RECs, HG treatment or receptor for advanced glycation end products activation by its ligand S100B induces the expression of TXNIP and inflammatory genes such as Cox2, VEGF-A and ICAM1. Nine modifications of histone $\mathrm{H} 3$ lysine and the $\mathrm{p} 38 \mathrm{MAPK}-\mathrm{NF}-\mathrm{\kappa B}$ signaling pathway are involved in TXNIP-induced inflammation. HG and advanced glycation end product receptor effects are impeded by silencing TXNIP, while stable overexpression of a cDNA for human TXNIP in endothelial cells elevates inflammation. Overexpression of TXNIP in endothelial cells abolishes $\mathrm{H} 3 \mathrm{~K} 9$ trimethylation, a gene inactivation marker, and increases $\mathrm{H} 3 \mathrm{~K} 9$ acetylation, a gene induction indicator, at the proximal Cox 2 promoter bearing the NF- $\kappa B$-binding site [79].

In addition to histone modifications, DNA methylation is also one of the mechanisms for the epigenetic control of gene expression, and aberrant DNA methylation patterns of $\mathrm{CpG}$ islands can influence normal transcriptional regulation [80]. The epigenetic regulation of DNA methylation in DR has seldom been reported. A recent study showed that the damage to the mitochondrial DNA (mtDNA) replication system, caused by the previous 3 months of PC, cannot be reversed by 3 months of GC. The replication enzymes remain downregulated, and the $\mathrm{D}$ loop region of the mtDNA continues to be damaged [81]. And the results of this study suggested that the possible reason for this damage could be continued hypermethylation of the $\mathrm{CpG}$ sites at the regulatory region of polymerase- $\gamma$, affecting its binding to the mtDNA, and compromising the transcriptional activity. These results 
strongly imply that the mtDNA replication system is impaired as a result of continued hypermethylation of polymerase- $\gamma$, and this continues even after the hyperglycemic insult is discontinued, DNA hypermethylation as one of the possible mechanisms is responsible for the failure to reverse mtDNA damage after reinstitution of GC and suggests an important role of DNA methylation in the metabolic memory phenomenon associated with the continued progression of DR [81].

The expression of miRNA has also changed in DR. miRNA-200b was found upregulated significantly in a type $1 \mathrm{DR}$ model. Oxidation resistance 1 , which attenuates oxidative stress markers and nitration of cellular proteins and ameliorates apoptosis induced by an oxidative stressor, 4-hydroxynonenal, is downregulated by transfection of an miR-200b mimic [82]. When rats treated with STZ were compared with untreated rats, changes in the expression of 37 miRNAs were detected in DR. Six of the confirmed altered miRNAs were differentially expressed over the course of STZ-induced diabetes. Levels of miRNA-182, miRNA-96, miRNA-211, miRNA-204, miRNA-183 and miRNA-124 were significantly increased during the progress of $\mathrm{DR}$, whereas miRNA-10b, miRNA-10a, miRNA-219-2-3p, miRNA144, miRNA-338 and miRNA-199a-3p were significantly decreased [83].

Another study [84] performed miRNA expression profiling in the retina and RECs of STZ-induced type 1 diabetes rats. They found that key NF- $\mathrm{B}$-responsive miRNAs (such as miRNA-146, miRNA-155, miRNA132 and miRNA-21) were upregulated in the diabetic RECs, and also that key VEGF-responsive miRNA17-5p, miRNA-18a, miRNA-20a, miRNA-21, miRNA31 and miRNA-133 and the p53-responsive miRNA-34 family were upregulated in both the retinas and RECs of the diabetic rats. NF- $\kappa \mathrm{B}$ is a key regulator of the immune response and plays an important role in the early pathogenesis of DR by triggering a proapoptotic program in retinal pericytes [85]. miRNAs, which are thought to be transcriptionally regulated by NF- $\kappa \mathrm{B}$ (miRNA-146, miRNA-155 and miRNA-21) [86-88], were also demonstrated to be upregulated in the diabetic RECs in rats [84]. This research confirmed that NF- $\kappa \mathrm{B}$ was able to directly activate miRNA-146 expression. Furthermore, overexpression of miRNA-146 inhibited interleukin-1 $\beta$ induced NF- $\mathrm{\kappa B}$ activation in RECs, forming a regulatory negative feedback loop to control NF- $\kappa \mathrm{B}$ and miRNA146 expression. Thus, overexpression of miRNA-146 may be exploited therapeutically by inhibition of NF- $\kappa B$ activation in DR [84]. VEGF, promoting angiogenesis and endothelial permeability, has been shown to be increased in early pathogenesis and to play important roles in DR $[89,90]$. The miRNA-34 family is a direct transcriptional target of p53 and contributes to p53-mediated cell cycle arrest, apoptosis and senescence [91]. Upregulation of VEGF- and p53-responsive miRNAs implicates miRNAs in mediating the proangiogenic or proapoptotic effects caused by VEGF and p53 in pathological changes of early DR [92]. In another study, reduced miRNA-200b and increased VEGF have been observed in human umbilical vein endothelial cells and bovine RECs treated with HG [92]. They also confirmed VEGF as a direct target, miRNA mimic treatments in vitro in endothelial cells and in vivo (intravitreal injection) could ameliorate increases in VEGF mRNA and protein levels induced by diabetes. Conversely, miRNA-200b antagomirs could increase VEGF production, thus providing further understanding of the role of this miRNA in the pathogenesis of DR [92]. Additional experimentation was performed in animal models: miR-200b mimic injection into the vitreous humor of diabetic mouse eyes resulted in locally decreased VEGF-A expression; in addition, knocking down miRNA-200b inhibits the diabetes-induced upregulation of p300 in the retina, implying crosstalk between two epigenetic mechanisms in DR [92]. Silva et al. [93] examined the role of miRNA-29b and its potential target RAX (an activator of the proapoptotic protein kinase $\mathrm{R}$ signaling pathway) in the apoptosis of retinal neurons related to the pathogenesis of DR. They observed that RAX and miR-29b were localized in the retinal ganglion cells and the cells of the inner nuclear layer of the retinas from normal and STZ-induced diabetic rats. Their results suggest that upregulation of miRNA-29b at the early stages of DR in this model may have protective effects against apoptosis of the retinal cells by the RNA-dependent protein kinase pathway. Therefore, intravitreal injections of key miRNAs such as miRNA-29b and miRNA-200b may be developed as translational approaches for the treatment of DR. In a more recent study, a decrease in miRNA-146a was observed in HG-treated endothelial cells from large vessels and retinal microvessels and in retinas from type 1 diabetes rats [94]. Moreover, this research demonstrated that the expression of fibronectin (an miRNA-146a target that can contribute to hypertrophy and fibrosis) was increased under HG conditions, due to decreases in miRNA-146a levels, which in turn was mediated by increases in the coactivator p300. 


\section{Conclusions}

Currently, studies have suggested an important role of epigenetic modifications in the continued progression of DR [74]. However, the understanding of the impact of epigenetic mechanisms in the pathogenesis in DR is very limited. With epigenetic mechanisms being extremely varied, including changes in DNA methylation, histone posttranslational modifications and miRNA expression, there is an extensive scope for further research in DR. Recent evidence shows that hyperglycemia can induce epigenetic changes to the chromatin structure via activation of various factors and signaling pathways. The specific key enzymes related to active and repressed chromatin states are involved in it, and epigenetic regulation of key inflammatory genes in retinas and RECs has been demonstrated. Well-defined cell and animal models treated with and without related interventions will further our understanding of epigenetic regulation and how to prevent $\mathrm{DR}$. The human epigenome project is expected to greatly enhance our understanding of epigenetic states under normal and disease conditions $[95,96]$. The generally accepted idea is that the histone code is reversible; therefore, greater understanding of the epigenetic basis of disease could enable the discovery of new therapeutic targets for the treatment of numerous human diseases, including DR. Epigenetic drugs such as inhibitors of DNA methylation, HATs and HDACs, and some histone demethylases are already being evaluated for cancer and other diseases $[34,97,98]$. We believe that available drugs could be used for their potential ability to alter epigenetic markers and become a therapeutic method for DR in the future.

\section{Acknowledgement}

The project was sponsored by grants from the National Natural Science Foundation of China (30973260 and 81371054).

\section{References}

$>1$ Kempen JH, O’Colmain BJ, Leske MC, Haffner SM, Klein R, Moss SE, et al; Eye Diseases Prevalence Research Group: The prevalence of diabetic retinopathy among adults in the United States. Arch Ophthalmol 2004;122: $552-563$.

-2 Saaddine JB, Honeycutt AA, Narayan KM, Zhang X, Klein R, Boyle JP: Projection of diabetic retinopathy and other major eye diseases among people with diabetes mellitus: United States, 2005-2050. Arch Ophthalmol 2008; 126:1740-1747.

$>3$ Liu L, Wu X, Liu L, Geng J, Yuan Z, Shan Z, et al: Prevalence of diabetic retinopathy in mainland China: a meta-analysis. PLoS One 2012;7:e45264.

$>4$ Bunce C, Wormald R: Leading causes of certification for blindness and partial sight in England and Wales. BMC Public Health 2006; 6:58.

5 Frank RN: Diabetic retinopathy. N Engl J Med 2004;350:48-58.

6 Curtis TM, Gardiner TA, Stitt AW: Microvascular lesions of diabetic retinopathy: clues towards understanding pathogenesis? Eye (Lond) 2009;23:1496-1508.

7 Villarroel M, Ciudin A, Hernández C, Simó R: Neurodegeneration: an early event of diabetic retinopathy. World J Diabetes 2010;1:57-64.

8 Barber AJ, Gardner TW, Abcouwer SF: The significance of vascular and neural apoptosis to the pathology of diabetic retinopathy. Invest Ophthalmol Vis Sci 2011;52:1156-1163.
-9 Kaiser N, Sasson S, Feener EP, BoukobzaVardi N, Higashi S, Moller DE, et al: Differential regulation of glucose transport and transporters by glucose in vascular endothelial and smooth muscle cells. Diabetes 1993;42:80-89.

10 Cheung N, Mitchell P, Wong TY: Diabetic retinopathy. Lancet 2010;376:124-136.

$\checkmark 11$ Yang Y, Hayden MR, Sowers S, Bagree SV, Sowers JR: Retinal redox stress and remodeling in cardiometabolic syndrome and diabe tes. Oxid Med Cell Longev 2010;3:392-403.

$\checkmark 12$ Adamis AP, Berman AJ: Immunological mechanisms in the pathogenesis of diabetic retinopathy. Semin Immunopathol 2008;30: 65-84.

13 Khan ZA, Chakrabarti S: Cellular signaling and potential new treatment targets in diabetic retinopathy. Exp Diabetes Res 2007;2007: 31867.

14 Wirostko B, Wong TY, Simo R: Vascular endothelial growth factor and diabetic complications. Prog Retin Eye Res 2008;27:608-621.

15 Kern TS: Contributions of inflammatory processes to the development of the early stages of diabetic retinopathy. Exp Diabetes Res 2007;2007:95103.

16 Li Q, Verma A, Han PY, Nakagawa T, Johnson RJ, Grant MB, et al: Diabetic eNOSknockout mice develop accelerated retinopathy. Invest Ophthalmol Vis Sci 2010;51:5240 5246.

17 Tang J, Kern TS: Inflammation in diabetic retinopathy. Prog Retin Eye Res 2011;30:343358 .
18 Nguyen TT, Alibrahim E, Islam FM, Klein R, Klein BE, Cotch MF, et al: Inflammatory, hemostatic, and other novel biomarkers for diabetic retinopathy: the multi-ethnic study of atherosclerosis. Diabetes Care 2009;32:17041709.

19 Ling C, Groop L: Epigenetics: a molecular link between environmental factors and type $2 \mathrm{di}-$ abetes. Diabetes 2009;58:2718-2725.

20 Litherland SA: Immunopathogenic interaction of environmental triggers and genetic susceptibility in diabetes: is epigenetics the missing link? Diabetes 2008;57:3184-3186.

21 Villeneuve LM, Natarajan R: Epigenetics of diabetic complications. Expert Rev Endocrinol Metab 2010;5:137-148.

22 The Diabetes Control and Complications Trial Research Group: The effect of intensive treatment of diabetes on the development and progression of long-term complications in insulin-dependent diabetes mellitus. N Engl J Med 1993;329:977-986.

23 DCCT Research Group and EDIC Research Group: Effect of intensive therapy on the microvascular complications of type 1 diabetes mellitus. JAMA 2002;287:2563-2569.

24 Wolffe AP, Guschin D: Review: chromatin structural features and targets that regulate transcription. J Struct Biol 2000;129:102-122.

-25 Nightingale KP, O’Neill LP, Turner BM: Histone modifications: signalling receptors and potential elements of a heritable epigenetic code. Curr Opin Genet Dev 2006;16:125-136.
Epigenetic Mechanisms in the Pathogenesis of DR
Ophthalmologica 2014;232:1-9 DOI: $10.1159 / 000357824$ 
26 Luger K, Mäder AW, Richmond RK, Sargent DF, Richmond TJ: Crystal structure of the nucleosome core particle at 2.8 Å resolution. $\mathrm{Na}$ ture 1997;389:251-260.

27 Roth SY, Denu JM, Allis CD: Histone acetyltransferases. Annu Rev Biochem 2001;70:81120.

28 Martin C, Zhang Y: The diverse functions of histone lysine methylation. Nat Rev Mol Cell Biol 2005;6:838-849.

29 Wade PA: Transcriptional control at regulatory checkpoints by histone deacetylases: molecular connections between cancer and chromatin. Hum Mol Genet 2001;10:693-698.

- 30 Bjerling P, Silverstein RA, Thon G, Caudy A, Grewal S, Ekwall K: Functional divergence between histone deacetylases in fission yeast by distinct cellular localization and in vivo specificity. Mol Cell Biol 2002;22:21702181, erratum in Mol Cell Biol 2002;22: 5257-5258.

-31 Miranda TB, Jones PA: DNA methylation: the nuts and bolts of repression. J Cell Physiol 2007;213:384-390.

-32 Vaissiere T, Sawan C, Herceg Z: Epigenetic interplay between histone modifications and DNA methylation in gene silencing. Mutat Res 2008;659:40-48.

-33 Murr R: Interplay between different epigenetic modifications and mechanisms. Adv Genet 2010;70:101-141.

34 Sharma S, Kelly TK, Jones PA: Epigenetics in cancer. Carcinogenesis 2010;31:27-36.

35 Saetrom P, Snove OJ, Rossi JJ: Epigenetics and microRNAs. Pediatr Res 2007;61:17R-23R.

-36 Bartel DP: MicroRNAs: genomics, biogenesis, mechanism, and function. Cell 2004;116: 281-297.

-37 Wutz A: RNA-mediated silencing mechanisms in mammalian cells. Prog Mol Biol Transl Sci 2011;101:351-376.

38 Bartel DP: MicroRNAs: target recognition and regulatory functions. Cell 2009;136:215-233.

-39 Croce CM, Calin GA: miRNAs, cancer, and stem cell division. Cell 2005;122:6-7.

-40 Kim VN, Han J, Siomi MC: Biogenesis of small RNAs in animals. Nat Rev Mol Cell Biol 2009; 10:126-139.

41 Gray SG, De Meyts P: Role of histone and transcription factor acetylation in diabetes pathogenesis. Diabetes Metab Res Rev 2005; 21:416-433.

42 Ito K, Hanazawa T, Tomita K, Barnes PJ, Adcock IM: Oxidative stress reduces histone deacetylase 2 activity and enhances IL- 8 gene expression: role of tyrosine nitration. Biochem Biophys Res Commun 2004;315:240245.

43 Vanden Berghe W, De Bosscher K, Boone E, Plaisance S, Haegeman G: The nuclear factorkappaB engages $\mathrm{CBP} / \mathrm{p} 300$ and histone acetyltransferase activity for transcriptional activation of the interleukin- 6 gene promoter. J Biol Chem 1999;274:32091-32098.
44 Reddy MA, Sahar S, Villeneuve LM, Lanting L, Natarajan R: Role of Src tyrosine kinase in the atherogenic effects of the 12/15-lipoxygenase pathway in vascular smooth muscle cells. Arterioscler Thromb Vasc Biol 2009;29: 387-393.

-45 Chen S, Feng B, George B, Chakrabarti R, Chen M, Chakrabarti S: Transcriptional coactivator p300 regulates glucose-induced gene expression in endothelial cells. Am J Physiol Endocrinol Metab 2010;298:E127E137.

46 Yun JM, Jialal I, Devaraj S: Epigenetic regulation of high glucose-induced proinflammatory cytokine production in monocytes by curcumin. J Nutr Biochem 2011;22:450-458.

$\checkmark 47$ Noh H, Oh EY, Seo JY, Yu MR, Kim YO, Ha $\mathrm{H}$, et al: Histone deacetylase- 2 is a key regulator of diabetes- and transforming growth factor-beta1-induced renal injury. Am J Physiol Renal Physiol 2009;297:F729-F739.

48 Chakrabarti SK, Francis J, Ziesmann SM, Garmey JC, Mirmira RG: Covalent histone modifications underlie the developmental regulation of insulin gene transcription in pancreatic beta cells. J Biol Chem 2003;278: 23617-23623.

- 49 Mutskov V, Raaka BM, Felsenfeld G, Gershengorn MC: The human insulin gene displays transcriptionally active epigenetic marks in islet-derived mesenchymal precursor cells in the absence of insulin expression. Stem Cells 2007;25:3223-3233.

50 Miao F, Wu X, Zhang L, Yuan YC, Riggs AD, et al: Genome-wide analysis of histone lysine methylation variations caused by diabetic conditions in human monocytes. J Biol Chem 2007;282:13854-13863.

51 Miao F, Smith DD, Zhang L, Min A, Feng W, Natarajan R: Lymphocytes from patients with type 1 diabetes display a distinct profile of chromatin histone $\mathrm{H} 3$ lysine 9 dimethylation: an epigenetic study in diabetes. Diabetes 2008;57:3189-3198.

52 Li Y, Reddy MA, Miao F, Shanmugam N, Yee JK, Hawkins D, et al: Role of the histone $\mathrm{H} 3$ lysine 4 methyltransferase, SET7/9, in the regulation of NF-kappaB-dependent inflammatory genes. Relevance to diabetes and inflammation. J Biol Chem 2008;283:26771-26781.

53 Brasacchio D, Okabe J, Tikellis C, Balcerczyk A, George P, Baker EK, et al: Hyperglycemia induces a dynamic cooperativity of histone methylase and demethylase enzymes associated with gene-activating epigenetic marks that coexist on the lysine tail. Diabetes 2009; 58:1229-1236.

54 El-Osta A, Brasacchio D, Yao D, Pocai A, Jones PL, Roeder RG, et al: Transient high glucose causes persistent epigenetic changes and altered gene expression during subsequent normoglycemia. J Exp Med 2008;205: 2409-2417.

- 55 Sun G, Reddy MA, Yuan H, Lanting L, Kato M, Natarajan R: Epigenetic histone methylation modulates fibrotic gene expression. J Am Soc Nephrol 2010;21:2069-2080.
56 Park JH, Stoffers DA, Nicholls RD, Simmons RA: Development of type 2 diabetes following intrauterine growth retardation in rats is associated with progressive epigenetic silencing of Pdx1. J Clin Invest 2008;118:2316-2324.

57 Ling C, Del Guerra S, Lupi R, Rönn T, Granhall C, Luthman $\mathrm{H}$, et al: Epigenetic regulation of PPARGC1A in human type 2 diabetic islets and effect on insulin secretion. Diabetologia 2008;51:615-622.

58 Kuroda A, Rauch TA, Todorov I, Ku HT, AlAbdullah IH, Kandeel F, et al: Insulin gene expression is regulated by DNA methylation. PLoS One 2009;4:e6953.

59 Morgan HD, Sutherland HG, Martin DI, Whitelaw E: Epigenetic inheritance at the agouti locus in the mouse. Nat Genet 1999;23: 314-318.

60 Muhonen P, Holthofer H: Epigenetic and microRNA-mediated regulation in diabetes. Nephrol Dial Transplant 2009;24:1088-1096.

61 Poy MN, Eliasson L, Krutzfeldt J, Kuwajima S, Ma X, Macdonald PE, et al: A pancreatic islet-specific microRNA regulates insulin secretion. Nature 2004;432:226-230.

$62 \mathrm{Mi}$ QS, He HZ, Dong Z, Isales C, Zhou L: MicroRNA deficiency in pancreatic islet cells exacerbates streptozotocin-induced murine autoimmune diabetes. Cell Cycle 2010;9:31273129.

63 Gallagher IJ, Scheele C, Keller P, Nielsen AR, Remenyi J, Fischer CP, et al: Integration of microRNA changes in vivo identifies novel molecular features of muscle insulin resistance in type 2 diabetes. Genome Med 2010;2: 9.

64 Heneghan HM, Miller N, Kerin MJ: Role of microRNAs in obesity and the metabolic syndrome. Obes Rev 2010;11:354-361.

65 Poy MN, Spranger M, Stoffel M: MicroRNAs and the regulation of glucose and lipid metabolism. Diabetes Obes Metab 2007;9(suppl 2):67-73.

66 Kato M, Arce L, Natarajan R: MicroRNAs and their role in progressive kidney diseases. Clin J Am Soc Nephrol 2009;4:1255-1266.

67 Kato M, Putta S, Wang M, Yuan H, Lanting L, Nair I, et al: TGF-beta activates Akt kinase through a microRNA-dependent amplifying circuit targeting PTEN. Nat Cell Biol 2009;11: 881-889.

68 Wang Q, Wang Y, Minto AW, Wang J, Shi Q, Li X, et al: MicroRNA-377 is up-regulated and can lead to increased fibronectin production in diabetic nephropathy. FASEB J 2008;22: 4126-4135.

69 Villeneuve LM, Natarajan R: The role of epigenetics in the pathology of diabetic complications. Am J Physiol Renal Physiol 2010; 299:F14-F25.

70 Zhong Q. Kowluru RA: Role of histone acetylation in the development of diabetic retinopathy and the metabolic memory phenomenon. J Cell Biochem 2010;110:1306-1313. 
71 Kowluru RA, Odenbach S: Effect of long-term administration of alpha-lipoic acid on retinal capillary cell death and the development of retinopathy in diabetic rats. Diabetes 2004;53: 3233-3238.

72 Kowluru RA, Atasi L, Ho YS: Role of mitochondrial superoxide dismutase in the development of diabetic retinopathy. Invest Ophthalmol Vis Sci 2006;47:1594-1599.

73 Kowluru RA, Kowluru V, Xiong Y, Ho YS: Overexpression of mitochondrial superoxide dismutase in mice protects the retina from diabetes-induced oxidative stress. Free Radic Biol Med 2006;41:1191-1196.

-74 Zhong Q, Kowluru RA: Epigenetic changes in mitochondrial superoxide dismutase in the retina and the development of diabetic retinopathy. Diabetes 2011;60:1304-1313.

-75 Zhong Q, Kowluru RA: Epigenetic modification of Sod2 in the development of diabetic retinopathy and in the metabolic memory: role of histone methylation. Invest Ophthalmol Vis Sci 2013;54:244-250.

-76 Forneris F, Binda C, Battaglioli E, Mattevi A: LSD1: oxidative chemistry for multifaceted functions in chromatin regulation. Trends Biochem Sci 2008;33:181-189.

77 Kowluru RA: Mitochondria damage in the pathogenesis of diabetic retinopathy and in the metabolic memory associated with its continued progression. Curr Med Chem 2013;20:3226-3233.

78 Zhong Q, Kowluru RA: Regulation of matrix metalloproteinase- 9 by epigenetic modifications and the development of diabetic retinopathy. Diabetes 2013;62:2559-2568.

-79 Perrone L, Devi TS, Hosoya K, Terasaki T, Singh LP: Thioredoxin interacting protein (TXNIP) induces inflammation through chromatin modification in retinal capillary endothelial cells under diabetic conditions. J Cell Physiol 2009;221:262-272.

80 Schinke C, et al: Aberrant DNA methylation in malignant melanoma. Melanoma Res 2010; 20:253-265.
81 Tewari S, Zhong Q, Santos JM, Kowluru RA: Mitochondria DNA replication and DNA methylation in the metabolic memory associated with continued progression of diabetic retinopathy. Invest Ophthalmol Vis Sci 2012; 53:4881-4888.

82 Murray AR, Chen Q, Takahashi Y, Zhou KK, Park K, Ma JX: MicroRNA-200b downregulates oxidation resistance 1 (Oxr1) expression in the retina of type 1 diabetes model. Invest Ophthalmol Vis Sci 2013;54:1689-1697.

$83 \mathrm{Wu} J \mathrm{H}$, Gao Y, Ren AJ, Zhao SH, Zhong M, Peng YJ, Shen W, Jing M, Liu L: Altered microRNA expression profiles in retinas with diabetic retinopathy. Ophthalmic Res 2012; 47:195-201.

84 Kovacs B, Lumayag S, Cowan C, Xu S: MicroRNAs in early diabetic retinopathy in streptozotocin-induced diabetic rats. Invest Ophthalmol Vis Sci 2011;52:4402-4409.

85 Kowluru RA, Koppolu P, Chakrabarti S, Chen S: Diabetes-induced activation of nuclear transcriptional factor in the retina, and its inhibition by antioxidants. Free Radic Res 2003; 37:1169-1180.

86 Taganov KD, Boldin MP, Chang KJ, Baltimore D: NF-kappaB-dependent induction of microRNA miR-146, an inhibitor targeted to signaling proteins of innate immune responses. Proc Natl Acad Sci USA 2006;103:1248112486.

87 Gatto G, Rossi A, Rossi D, Kroening S, Bonatti S, Mallardo M: Epstein-Barr virus latent membrane protein 1 trans-activates miR-155 transcription through the NF-kappaB path way. Nucleic Acids Res 2008;36:6608-6619.

88 Sheedy FJ, Palsson-McDermott E, Hennessy EJ, Martin C, O'Leary JJ, Ruan Q, et al: Negative regulation of TLR4 via targeting of the proinflammatory tumor suppressor PDCD4 by the microRNA miR-21. Nat Immunol 2010;11:141-147.

89 Sone H, Kawakami Y, Okuda Y, Sekine Y, Honmura S, Matsuo K, et al: Ocular vascular endothelial growth factor levels in diabetic rats are elevated before observable retinal proliferative changes. Diabetologia 1997;40:726730 .
90 Antonetti DA, Barber AJ, Hollinger LA, Wolpert EB, Gardner TW: Vascular endothelial growth factor induces rapid phosphorylation of tight junction proteins occludin and zonula occluden 1 . A potential mechanism for vascular permeability in diabetic retinopathy and tumors. J Biol Chem 1999;274:23463-23467.

$91 \mathrm{He}$ L, He X, Lowe SW, Hannon GJ: microRNAs join the p53 network - another piece in the tumour-suppression puzzle. Nat Rev Cancer 2007;7:819-822.

92 McArthur K, Feng B, Wu Y, Chen S, Chakrabarti S: MicroRNA-200b regulates vascular endothelial growth factor-mediated alterations in diabetic retinopathy. Diabetes 2011; 60:1314-1323.

93 Silva VA, Polesskaya A, Sousa TA, Corrêa VM, André ND, Reis RI, et al: Expression and cellular localization of microRNA-29b and RAX, an activator of the RNA-dependent protein kinase (PKR), in the retina of streptozotocin-induced diabetic rats. Mol Vis 2011; 17:2228-2240.

94 Feng B, Chen S, McArthur K, Wu Y, Sen S, Ding Q, Feldman RD, Chakrabarti S: miR-146a-mediated extracellular matrix protein production in chronic diabetes complications. Diabetes 2011;60:2975-2984.

95 American Association for Cancer Research Human Epigenome Task Force; European Union, Network of Excellence, Scientific Advisory Board: Moving AHEAD with an international human epigenome project. Nature 2008;454:711-715.

96 Qiu J: Epigenetics: unfinished symphony. Nature 2006;441:143-145.

97 Aggarwal BB, Harikumar KB: Potential therapeutic effects of curcumin, the anti-inflammatory agent, against neurodegenerative, cardiovascular, pulmonary, metabolic, autoimmune and neoplastic diseases. Int J Biochem Cell Biol 2009;41:40-59.

98 Shi Y: Histone lysine demethylases: emerging roles in development, physiology and disease. Nat Rev Genet 2007;8:829-833.
Epigenetic Mechanisms in the Pathogenesis of DR
Ophthalmologica 2014;232:1-9 DOI: $10.1159 / 000357824$ 